11 | 2018

Écrire le fleuve Congo après Conrad

\title{
Heart of Darkness, exposition
}

\section{Christian Tundula}

\section{OpenEdition}

\section{Journals}

Édition électronique

URL : https://journals.openedition.org/coma/2878

DOI : $10.4000 /$ coma. 2878

ISSN : 2275-1742

\section{Éditeur}

Institut des textes \& manuscrits modernes (ITEM)

\section{Référence électronique}

Christian Tundula, « Heart of Darkness, exposition », Continents manuscrits [En ligne], 11 | 2018, mis en ligne le 15 octobre 2018, consulté le 12 janvier 2023. URL : http://journals.openedition.org/coma/2878 ; DOI : https://doi.org/10.4000/coma.2878

Ce document a été généré automatiquement le 12 janvier 2023.

\section{cc) (i) $(9)$}

Creative Commons - Attribution - Pas d'Utilisation Commerciale - Pas de Modification 4.0 International - CC BY-NC-ND 4.0

https://creativecommons.org/licenses/by-nc-nd/4.0/ 


\title{
Heart of Darkness, exposition
}

\author{
Christian Tundula
}

\section{Conrad, une source d'inspiration}

1 À cette table, interviennent beaucoup de littéraires, de chercheurs, de gens qui ont passé du temps à étudier Conrad. Pour moi, en revanche, c'est par hasard qu'en 2016 j'ai découvert l'œuvre de Joseph Conrad, mais pas aussi profondément que ça. Je vais commencer à répondre à la première question qui nous a été posée : comment dans mon travail, Conrad a-t-il pu être une source d'inspiration ou un objet de questionnement?

2 Je suis né au Congo, à Kinshasa, et mon travail, qui est autobiographique, porte sur le Congo et sur Kinshasa. Quand je regarde le récit et l'expérience personnelle de Conrad qui se déroulent au cœur du Congo, je m'y plonge moi aussi, et cela m'incite à me réapproprier cette histoire-là en tant qu'esthéticien congolais, et à réécrire ma propre histoire.

3 Ma série photographique Heart of Darkness, que j'avais nommée « Mémoire », est fondée sur ma propre expérience de jeune homme, lorsque j'ai dû identifier le corps de mon cousin le long du fleuve Congo. Je me suis inspiré de ma mémoire personnelle. Ce paramètre explique le cadre visuel de mon obsession pour l'enquête policière, et m'a permis de construire un parallèle avec le fleuve Congo en tant que lieu des ténèbres. Au centre de mes photographies, il y a l'idée qu'il existe des différences entre ceux qu'on appelle les «agents de l'ordre » et ceux qu'on appelle Shegués (« enfants des rues») ou Romains («les passeurs»). Joseph Conrad a fondé lui aussi son récit sur sa propre expérience au Congo : il s'est inspiré de son journal pour écrire l'obsession du narrateur Marlow pour le trafiquant d'ivoire Kurtz. Marlow enquête sur le mystérieux Kurtz, sur sa sauvagerie et sur son humanité, en Afrique, le long du fleuve Congo. Et le roman repose lui aussi sur l'idée centrale selon laquelle il y a une différence entre les gens civilisés et ceux qui sont décrits comme des sauvages. Mon travail poursuit le même questionnement : pour moi, ceux qu'on appelle «agents de l'ordre » ne sont pas des 
agents de l'ordre. Il se crée une confusion entre ces agents de l'ordre et ceux qu'on appelle Shegués ou Romains - les enfants des rues ou les passeurs qui font les traversées ${ }^{1}$.

Heart of Darkness est un projet photographique en noir et blanc où je construis des séries de portraits photographiques à partir d'anciens clichés que je n'avais jamais explorés ni exploités auparavant. Mes œuvres sont donc un voyage autobiographique dans l'abîme de mon espace psychique, sous la chair, au cœur des ténèbres, où mon fantôme s'égare entre abstraction et réalité, au-delà du domaine du photojournalisme.

\section{Heart of Darkness}

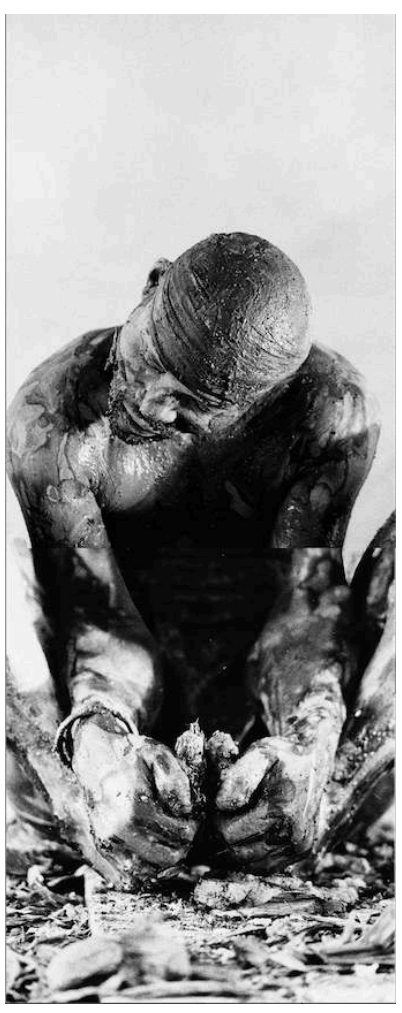

Technique mixte : photo, collage, encre de chine...

Autorisation de l'artiste

Lorsque vous pénétrez dans mes images, mon travail vise à éveiller l'empathie pour ces portraits, ces personnes, que tout le monde possède grâce à son propre fantôme : le passé opprimé ou le cœur des ténèbres de chacun d'entre nous. Il appartient à chacun d'entre nous de décider quoi en faire. Pour moi, c'est l'acte d'affronter et d'embrasser le fantôme - le fantôme est mon cousin décédé - qui me conduit à me racheter ou à me libérer du passé, à visualiser le futur, et à prendre le contrôle de ma vie, malgré la situation dans laquelle je suis né.

6 Je suis né en 1978, je fais donc partie de la génération qu'on appelle celle du Mobutisme et qui a grandi avec le concept du débrouillatisme suivant «l'article $15^{2}$ », selon lequel chacun devait se prendre en charge. J'ai donc eu la chance de me retrouver assistant photographe à l'âge de 20 ans tandis que mon cousin, qui était de mon âge, n'a pas trouvé de travail et a choisi un chemin dangereux, celui de passeur illégal de produits au port du Beach Ngobila ${ }^{3}$, l'un des ports fluviaux les plus importants de Kinshasa, pour gagner de l'argent. Il transportait des produits de bateau et essayait de les faire passer sans la douane. C'était la façon pour les commerçants d'éviter les taxes et de faire plus 
de profits. Mais, malheureusement, un jour il a été poursuivi, lui et ses copains, et chassé par un policier. Par peur, il glissa et tomba dans le fleuve. Le cadavre s'est égaré dans le fond du Congo boueux pendant trois jours, jusqu'à ce qu'il remonte à la surface, gonflé par le méthane, l'hydrogène sulfuré et le dioxyde de carbone produit par les bactéries dans l'intestin et la cavité thoracique. J'ai été appelé par la police pour identifier le cadavre parce que "mon frère " portait les mêmes vêtements que moi. Je n'ai pu l'identifier que grâce à la déformation qu'il avait depuis le plus jeune âge sur sa jambe: c'est pourquoi dans mes photographies, je travaille sur les proportions humaines - dans un centre d'anatomie à l'Académie des Beaux Arts - sur le rapport entre les corps et les objets.

7 Au-delà de sa dimension autobiographique, mon travail est aussi une interprétation, une préfiguration, une imagination qui imprègne chaque moment de mon autobiographie, comme l'histoire de l'écrivain Joseph Conrad. La plupart du temps, cette préfiguration fonctionne au niveau de l'imagerie, qui est toujours sombre. Je ne suis pas un littéraire, mais j'ai essayé de réécrire l'histoire que j'ai vécue avec mon cousin d'une façon visuelle. Je me suis également inspiré de ses mémoires d'enfance ainsi que des quelques récits de ses amis Romains, lorsqu'ils nous ont relaté les faits, à la famille et à moi, et qu'ils nous ont raconté ce qui s'était passé ce jour-là à Beach Ngobila, sur le fleuve Congo. Comme Conrad, visiteur au Congo, je me suis inspiré du récit des Romains et de ma mémoire, je me les suis réappropriés : je connais le fleuve, le Congo de mon enfance. Mais je dois faire un parallèle entre ma mémoire et les récits qui racontent les faits douloureux du Beach Ngobila.

8 Je voudrais terminer sur le fait que j'ai toujours porté un grand intérêt à la vie quotidienne dans le quartier urbain ou j'ai grandi à Kinshasa. Je m'en inspire toujours et me passionne encore pour l'amélioration, la créativité et l'indépendance réelle de mon pays et de mon peuple face à l'impérialisme, au capitalisme et à l'esclavagisme modernes.

Je remercie les organisateurs de cette table ronde.

\section{Mon travail technique}

10 Heart of Darkness est un travail photographique noir sur blanc. Je l'ai réalisé avec mon vieil appareil photo Nikon $\mathrm{F} 4$, avec mon objectif $35 \mathrm{~mm}$. Je ne sais pas si c'est un travail de photographie ou de collage ou une installation photographique. Mais j'utilise mes photographies comme moyen de m'exprimer. Elles deviennent des matériaux que je peux manipuler manuellement pour leur donner la forme que je veux, selon la façon dont j'imagine ou interprète chaque image. J'ai ainsi reconstruit des séries de photographies : après les avoir imprimées, je les ai coupées et collées manuellement comme un collage. Après les avoir rapiécées, j'ai utilisé de l'encre de Chine que j'ai appliquée sur certaines parties en guise de finitions.

\section{Mon approche artistique}

11 En 2006, j'ai produit des portraits en noir et blanc intitulés «objets corporels », ce travail était l'expression de mon sentiment sur ma vie mais aussi sur ce qui se passait autour de moi, en particulier dans mon pays. Cette inspiration m'est venue en 
travaillant dans une grande salle d'atelier de sculpture à l'école où j'ai effectué mes études d'art visuel, à l'Académie des beaux-arts de Kinshasa. J'étais entouré de centaines de moules abandonnés par les étudiants, des parties de sculptures brisées, et j'étais très inspiré par les différentes formes et textures de ces moules. C'est ainsi que j'ai réalisé une série de photographies en noir et blanc dans lesquelles j'ai essayé de photographier le corps d'un de mes amis modèle : après avoir appliqué de l'argile et de la boue et attendu que le corps sèche, j'ai trouvé que cela ressemblait à une sculpture. J'avais alors réalisé une très bonne série photographique d'un corps devenu d'objet, déstructuré, déformé, déshumanisé : ce résultat était dû à la façon dont j'avais photographié séquentiellement le corps de mon sujet.

\section{Heart of Darkness}

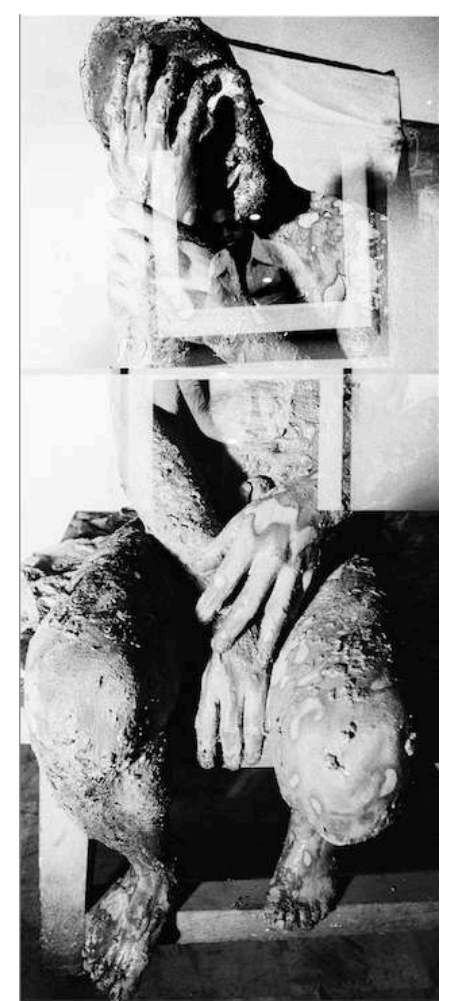

Technique mixte : photo, collage, encre de chine...

Autorisation de l'artiste

Dix ans plus tard, j'ai eu l'idée d'une reconstitution photographique fondée sur les techniques de la découpe, du collage et de l'application de l'encre de chine sur mes photographies. C'est ainsi que ma série Heart of Darkness a commencé. Les séries de portraits que j'ai faites, et celles qui sont en cours, sont le résultat d'un travail continu depuis 2006. Mes photographies sont un requiem pour mon frère, un rappel constant du chemin qu'il a pris et une réflexion sur la façon dont une décision peut conduire à des futurs complètement différents. Parce que mon travail concerne des souvenirs. Des souvenirs personnels, je les dédie à « mon frère ». 


\section{NOTES}

1. Christian Tundula nous explique par la suite en quoi consiste ce travail de passeur et auquel sont obligés nombre de jeunes gens pour gagner leur vie.

2. Expression du Congo-Kinshasa en cours depuis les années soixante, quand le Sud-Kasaï prit son indépendance, et se référant à un article imaginaire de la Constitution du nouvel État, selon lequel chacun devait se débrouiller. Cette expression s'est popularisée pour expliquer la débrouillardise et la corruption.

3. Beach Ngobila est le port d'embarquement et de débarquement des passagers qui traversent le fleuve Congo entre Kinshasa et Brazzaville.

\section{RÉSUMÉS}

En tant que photographe, Christian Tundula s'interroge sur son lien avec l'œuvre de Conrad.

As a photograph, Christian Tundula asks himself about his link with Joseph Conrad.

\section{AUTEUR}

\section{CHRISTIAN TUNDULA}

Photographe, CHRISTIAN TUNDULA vit et travaille en RDC et à Bruges. Après des études de communication visuelle à l'Académie des beaux-arts de Kinshasa et à l'École supérieure des arts décoratifs de Strasbourg, il a été en résidence d'artiste à la Fondation Blachère et a participé aux Rencontres de la photographie panafricaine de Johannesburg. 